\title{
Blink Rate Behavioral Pattern Changes in Patients Affected by Acute Stroke: A Cross-Sectional Study
}

\author{
Belvisi D*, Li Voti P, Nardella A, Ferrazzano G, Damulin IV, Tinelli E, Toschi N and Spallone A \\ Department of Clinical Neurosciences, Italy
}

*Corresponding author: Daniele Belvisi, Department of Clinical Neurosciences, Section of Neurosurgery, Neurological Centre of Latium-Neuromed, Rome, Italy.

Received Date: September 26, 2019

Published Date: September 30, 2019

\begin{abstract}
Spontaneous eye blinking is a synchronous, bilateral, short-term closure of the eyelids and can be quantified in terms of blink rate, the number of blinks per minute. A number of studies in healthy subjects have demonstrated that blink rate changes according to behavioral tasks (e.g. reading, writing, speaking). Blink rate and its task-induced modulation therefore represent a simple and reliable biomarker of the activity of several brain areas and/or connections. Stroke is a major worldwide source of disability: the high risk of death as well as long-term disability lead to a significant physical, psychological and financial burden for patients as well as for caregivers. The aim of this pilot cross-sectional study was to investigate the possible modulation of blink rate in patients affected by acute cerebrovascular events. We enrolled 20 consecutive patients with acute stroke (mean age \pm standard deviation:66.5 \pm 10.4 ; males:14) and 15 sex- and age-matched (mean age \pm standard deviation: 64.3 \pm 10.4 ; males:9) healthy subjects as controls. We measured the blink rate during 4 different conditions. Our findings showed that blink rate at rest was significantly higher in patients with stroke (examined within 24 hours after the ischemic event) than in healthy controls. Also, in both groups blink rate was significantly lower during writing and reading in comparison with blink rate at rest. Our findings showed that different behavioral patterns of spontaneous blinking are abnormal in the early phase of post-stroke course. In further studies we would try to use spontaneous blinking as a predictor of the residual functionality in patients with acute stroke.
\end{abstract}

Keywords: Stroke; Blink rate; Reading; Writing; Speaking

Abbreviations: NIHSS: NIH Stroke Scale/Score; MoCA: Montreal Cognitive Assessment

\section{Introduction}

Spontaneous eye blinking is a synchronous, bilateral, shortterm closure of the eyelids [1] and can be quantified in terms of blink rate, i.e. the number of blinks per minute [2]. While the cerebral circuits underlying spontaneous blinking have not yet been identified, it is thought that the trigeminal-facial complex may represent the main generator of this phenomenon [3] and that central dopaminergic tone exerts a key influence on this circuit [4]. A number of studies in healthy subjects have demonstrated that blink rate changes according to behavioral tasks (e.g. reading, writing, speaking), hence suggesting that it may be modulated by an interplay of several cortical regions (i.e. a "spontaneous blinking network") [5]. Blink rate and its task-induced modulation therefore represent a simple and reliable biomarker of the activity of several brain areas and/or connections. Indeed, previous studies have demonstrated changes in these markers in different neurological neurodegenerative conditions, such as Parkinson's disease [6], PSP [7] and blepharospasm [8,9].

Stroke is a major worldwide source of disability. The high risk of death as well as long-term disability lead to a significant physical, psychological and financial burden for patients as well as for caregivers [10]. Recent neuroimaging studies have demonstrated that loss of function after stroke is associated with abnormal connectivity in a large number of integrative networks beyond lesion territory [11]. Investigating blink rate patterns in patients with acute stroke may, therefore, provide new information about residual brain functionality and possibly provide markers which can be predictive of functional recovery.

The aim of this pilot cross-sectional study was to investigate the possible modulation of blink rate in patients affected by acute cerebrovascular events as well as in control subjects matched for 
age and sex during the execution of specific tasks such as writing, reading and speaking.

\section{Methods}

\section{Subjects}

We enrolled 20 consecutive patients with acute stroke (mean age \pm standard deviation: $66.5 \pm 10.4$; males: 14$)$ and 15 sex- and age-matched (mean age \pm standard deviation: 64.3 \pm 10.4 ; males: 9) healthy subjects as controls. All patients were recruited at the Department of Clinical Neurosciences, Section of Neurology, Neurological Centre of Latium-Neuromed, Rome. Information about clinical characteristics, disease course and family history were gathered through a face-to-face interview with expert neurologists and neuropsychologists. All patients were studied within 24 hours after acute stroke. Stroke severity was quantified using the NIH Stroke Scale/Score (NIHSS). We excluded subjects with a history of exposure to dopamine receptor blocking agents within 6 months prior to enrolment, as well as patients with previous and current ophthalmological disorders or who used contact lenses. Patients with significant cognitive disturbances (as defined by achieving a score lower than 26 in the Montreal Cognitive Assessment [MoCA]) were also excluded. The experimental procedure was approved by the institutional review boards and was conducted in accordance with the Declaration of Helsinki. Written informed consent was obtained from every participant.

\section{Clinical assessment}

Participants were videotaped. We measured the blink rate during 4 different conditions:

1. At rest

2. While writing a sentence on paper

3. During a conversation based on a simple topic (speaking task) that did not imply any emotional impact or memory recall

4. While reading aloud. Task order was randomized between patients

Each video recording lasted 60 seconds, with an interval of 10 seconds between each of the 4 recordings. All video recordings were performed in a noiseless room with artificial light. The camera was directly facing the patients at a distance of 2 meters. Two independent stroke specialists (PLV, AN) reviewed the videotapes and measured the number of blinks in each recording. During video recording evaluation, spontaneous blinking was measured by calculating the blink rate and was expressed as number of blinks per minute. Demographic and clinical data of patients with acute stroke are reported in Table 1.

Table 1: Demographic and clinical data of patients with acute stroke. MoCA: Montreal Cognitive Assessment. NIHSS: NIH Stroke Scale/Score.

\begin{tabular}{|c|c|c|c|c|c|c|}
\hline Patient & Age & Gender (M:1; F: 0) & MoCA score & NIHSS score & $\begin{array}{l}\text { Cerebral emisphere } \\
\text { (right:1; left: 0) }\end{array}$ & Stroke location \\
\hline 1 & 69 & 1 & $30 / 30$ & 4 & 1 & Temporo-occipital white matter \\
\hline 2 & 72 & 1 & $29 / 30$ & 3 & 1 & Thalamus \\
\hline 3 & 64 & 1 & $27 / 30$ & 1 & 0 & Thalamus \\
\hline 4 & 57 & 0 & $28 / 30$ & 3 & 0 & Fronto-parietal cortex \\
\hline 5 & 75 & 1 & $30 / 30$ & 1 & 0 & Internal capsule posterior limb \\
\hline 6 & 58 & 1 & $30 / 30$ & 6 & 0 & Capsular-lenticular region \\
\hline 7 & 84 & 0 & $26 / 30$ & 2 & 1 & Capsular-lenticular region \\
\hline 8 & 46 & 0 & $30 / 30$ & 1 & 1 & Hippocampus \\
\hline 9 & 82 & 1 & $30 / 30$ & 6 & 0 & Corona radiata \\
\hline 10 & 81 & 1 & $27 / 30$ & 2 & 0 & Centrum semiovale \\
\hline 11 & 72 & 1 & $29 / 30$ & 1 & 0 & Corona radiata \\
\hline 12 & 64 & 0 & $30 / 30$ & 1 & 1 & Frontal white matter \\
\hline 13 & 70 & 0 & $28 / 30$ & 0 & 1 & Periventricular region \\
\hline 14 & 60 & 1 & $30 / 30$ & 3 & 0 & Fronto-parietal cortex \\
\hline 15 & 70 & 1 & $28 / 30$ & 3 & 1 & Periventricular/Internal capsule \\
\hline 16 & 60 & 1 & $30 / 30$ & 1 & 1 & Corona radiata \\
\hline 17 & 45 & 1 & $30 / 30$ & 1 & 1 & Hippocampus \\
\hline 18 & 64 & 1 & $28 / 30$ & 2 & 1 & Internal capsule posterior limb \\
\hline 19 & 69 & 0 & $26 / 30$ & 5 & 1 & Corona radiata \\
\hline 20 & 69 & 1 & $30 / 30$ & 3 & 0 & Periventricular/Corona radiata \\
\hline
\end{tabular}

\section{Radiological assessment}

A conventional MRI study was performed. MR images were acquired with a 1.5T magnet (GE-Excite). All patients were investigated during a single session with the following sequences and parameters T2 fast spin-echo (TR: $2150 \mathrm{~ms}$, TE: $120 \mathrm{~ms}$; matrix:
256×256; thickness: $5 \mathrm{~mm}$; 22 axial slices); T2 gradient-echo (TR: 520ms, TE: 15ms; FA: 10; matrix: 256 $\times 192$; thickness: $5 \mathrm{~mm} ; 22$ axial slices); T2 fast spin-echo (TR: 4000ms, TE: 98ms; matrix: $384 \times 192$; thickness: $5 \mathrm{~mm}$; 24 coronal slices); T1 spin-echo (TR: 550ms, TE: 9ms; matrix: 320×256; thickness: 5mm; 22 axial slices); 
T1 spin-echo (TR: 460ms, TE: 9ms; matrix: 320×192; thickness: $5 \mathrm{~mm}$; 24 sagittal slices); T2 fluid attenuated inversion recovery (TR: $8000 \mathrm{~ms}$, TE: $82 \mathrm{~ms}$; TI: $2000 \mathrm{~ms}$; matrix: $320 \times 192$; thickness: 5mm; 22 axial slices); Diffusion epi (TR: 6000ms, TE: 75ms; matrix: $96 \times 96$; thickness: $5 \mathrm{~mm} ; 24$ axial slices; $b=0$ and $1000 \mathrm{sec} /$ $\mathrm{mm} 2$ ); TOF 3D time of flight (TR: 23ms, TE: 2.8ms; FA: 20; matrix: $320 \times 224$; thickness: $1 \mathrm{~mm}$; 168 axial slices).

Maps of apparent diffusion coefficient (ADC) were generated from the diffusion-weighted acquisitions. We enrolled all patients showed an hyperintensity in diffusion weighted maps, corresponding to with reduced values of the apparent diffusion coefficient.

Lesions size was measured on DWI axial sequences (maximum size: $1.7 \mathrm{~cm})$.

\section{Statistical analysis}

Statistical analysis was performed using the SPSS software (v. 24.0). To evaluate blink rate at rest we used a between-group oneway ANOVA with GROUP (patients vs controls) as the main factor. To evaluate any changes in the blink rate during the execution of the cognitive task, we performed a two-way, repeated measures ANOVA with within-factor GROUP (two levels: patients vs HS) and between-factor TASK (three levels: writing, reading and speaking), which also included a GROUPXTASK interaction term. Whenever a significant factor effect was detected, pairwise comparisons were conducted and corrected for multiple comparisons using Bonferroni's technique. A p value $<0.05$ (corrected) was considered statistically significant. Spearman's correlation coefficient was used to investigate possible correlations between either blink rate at rest or blink rate modulation and demographic and clinical variables, including age, gender, NIHSS score, MoCA score, body side involved).

\section{Results}

Blink rate at rest in patients with stroke and healthy controls. The blink rate at rest was $21.3 \pm 13.5$ (mean \pm SD) in patients with acute stroke and in 10.6 \pm 5.8 (mean \pm SD) healthy controls (Figure 1). The between-group one-way ANOVA showed that spontaneous BR at rest was significantly higher in patients with stroke as compared to healthy controls ( $\mathrm{F}=6.62 ; \mathrm{p}=0.01$ ) (Figure 1$)$.

\section{Blink rate at rest}

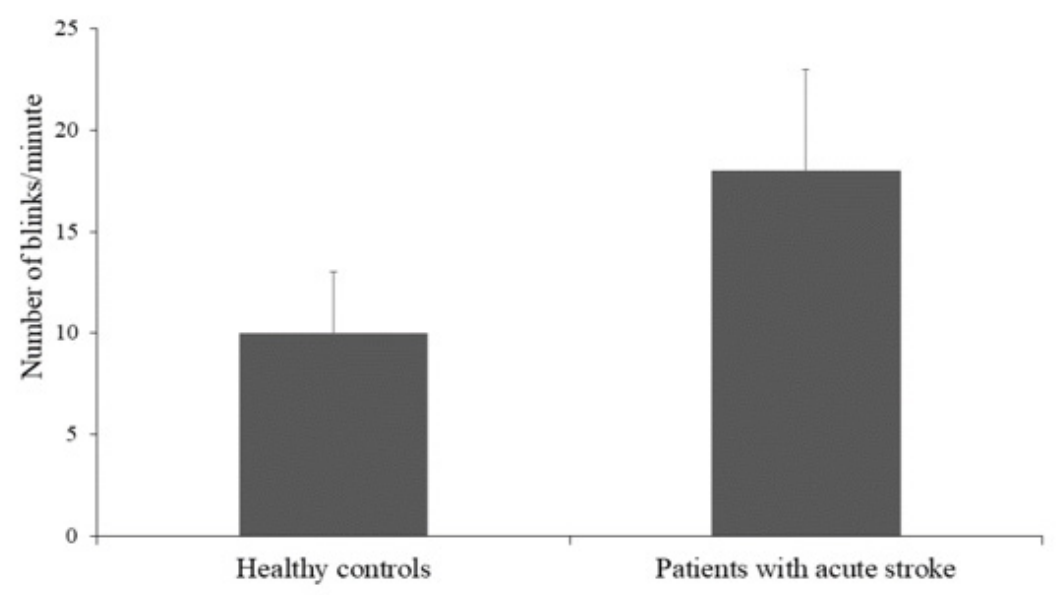

Figure 1: Blink rate at rest in patients with acute stroke and healthy controls.

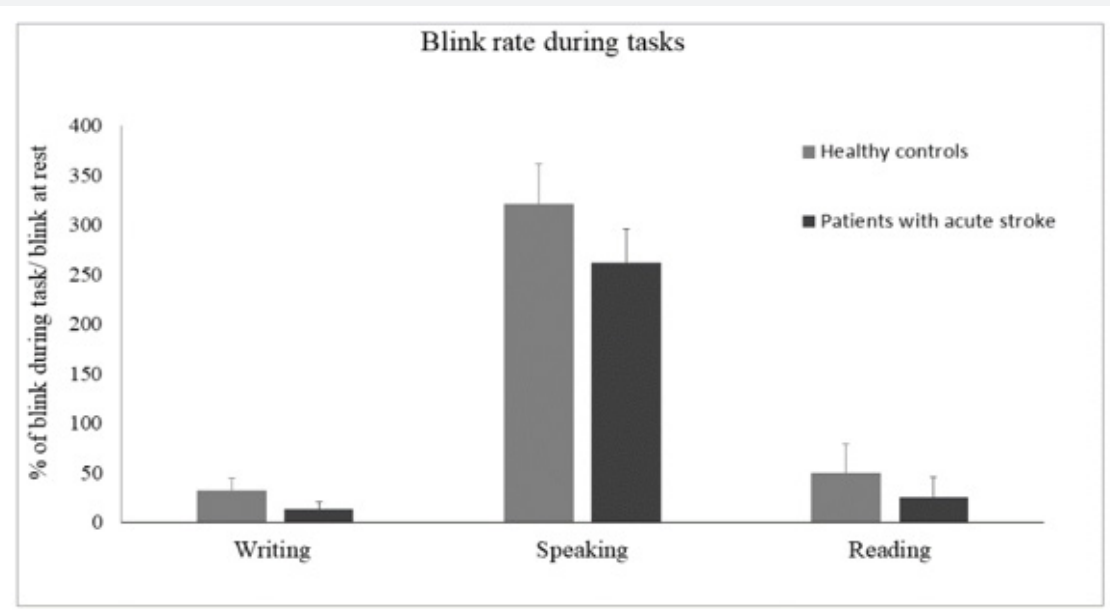

Figure 2: Blink rate percentage changes during writing, speaking and reading in patients with acute stroke and healthy controls. 
Changes in blink rate during speaking, reading and writing: patients with stroke vs healthy controls:

The between group repeated measures ANOVA investigating the changes of BR during tasks in patients and healthy controls showed a significant effect of the factor GROUP ( $F=5.27 ; p=0.02)$ as well as of the factor TASK ( $\mathrm{F}=42.2 ; \mathrm{p}=0.0001)$ and of the GROUPXTASK interaction ( $\mathrm{p}=0.04$ ) (Figure 2). In detail, writing and reading both induced a significant reduction of blink rate when compared with blink rate at rest, and post hoc analysis revealed that blink rate was more reduced during writing $(\mathrm{p}=0.003)$ and reading $(\mathrm{P}=0.003)$ in patients than in controls. Speaking induced a similar increase of blink rate in both groups ( $\mathrm{p}=0.06$ ) (Figure 2).

No correlations between blink rate and demographic/clinical variables were found.

\section{Discussion}

Our findings showed that blink rate at rest was significantly higher in patients with stroke (examined within 24 hours after the ischemic event) than in healthy controls. Also, in both groups blink rate was significantly lower during writing and reading in comparison with blink rate at rest. Patients with stroke, however, showed a more pronounced reduction of the blink rate during writing and reading when compared with healthy controls. Conversely, speaking induced a similar increase of the blink rate in comparison with blink rate at rest in the two groups. Given that the video recording was performed in a comfortable environment with controlled light intensity, we assumed that environmental conditions did not affect spontaneous blinking. Also, task order randomization ensured that different levels of attention did not systematically influence our findings. Given that cortical dopamine levels may alter the blink rate, we excluded any subject treated with dopaminergic agonists and blockers.

To our knowledge, only one previous study investigated spontaneous blinking in patients with acute stroke [12], where the authors found no overall difference in BR across the whole stroke population (although differences were found in subjects with frontal infarcts). However, blink rate was examined during a free conversation on trivial subjects requiring no emotional or cognitive effort as well as during mental arithmetic. Therefore, the present study provides the first evaluation of the blink rate at rest in patients with acute stroke. Furthermore, we only included subjects who had an acute stroke in the previous 24 hours while Anagnostou et al. [12] included patients within 2 days after symptom onset. It is therefore possible that in patients with stroke blink rate that tends to normalize in the early phase of post-stroke course after a transient change. Give that a longitudinal assessment was lacking in both studies it is, however, not possible to establish to exact duration of the increase of blink rate after acute stroke.

Although the brain circuitry underlying spontaneous blinking is still not fully understood, evidence in animal models suggests that trigeminal-facial complex, under the influence of dopaminergic descending pathways, plays a key role $[3,13]$. None of our patients had brainstem lesions. It is, therefore, likely that the higher spontaneous blinking of patients with stroke may depend on a diaschisis of descending pathways due to acute stroke. Our findings need to be confirmed in a future study involving a larger population of patients with stroke.

A further finding of our study was that patients with stroke had a more pronounced reduction of blink rate during writing and reading than healthy controls, while speaking induced a similar increase of blink rate in patients with stroke and healthy controls. Previous MRI studies have shown that in healthy subjects several cerebral areas are activated during writing [14,15] and reading [16] suggesting that a complex brain network underlies these behavioral tasks. For instance, the blink rate reduction induced by writing and reading is thought to depend on occipital inhibitory projections that exert a direct and indirect modulation on the trigemino-facial circuits in the brainstem [17]. The excessive reduction of blink rate during writing and reading observed in patients with stroke in comparison to healthy controls may depend either on brain early compensatory mechanisms in response to the acute damage or on a stroke-induced instability of cortical networks.

Overall, our exploratory cross-sectional study showed that the different behavioral patterns of spontaneous blinking are abnormal in the early phase of post-stroke course. Our purpose is to extend the present findings by performing a longitudinal controlled clinical-radiological study in a larger population of patients affected by acute stroke. This design will allow to clarify whether spontaneous blinking may represent a predictor of the residual functionality of the integrative circuits in patients with acute stroke and to establish the relationship between infarct locations and spontaneous blinking abnormalities.

\section{Author contributions statement}

- $\quad$ Conception and design of the study: DB, PLV

- $\quad$ Acquisition of data: PLV, AN, GF, ET

- $\quad$ Analysis and interpretation of data: PLV, AN, DB, NT

- $\quad$ Drafting the article: DB, PLV

- Critical revision for important intellectual content: DB, PLV, AS, NT

- $\quad$ Final approval of the version to be submitted: AS

\section{Acknowledgements}

- $\quad$ Formatting of funding sources: This research did not receive any specific grant from funding agencies in the public, commercial, or not-for-profit sectors.

- $\quad$ Nothing to declare.

\section{Conflict of interest disclosure}

- The authors declare that there is no conflict of interest regarding the publication of this paper.

- The authors declare that the submitted work was not 
carried out in the presence of any personal, professional or financial relationships that could potentially be construed as a conflict of interest.

\section{References}

1. Karson CN (1989) Blinking. Bull Soc Belge Ophtalmol 237: 443-457.

2. McMonnie CW (2010) Blinking mechanisms. In: Encyclopedia of the Eye: 202-208.

3. Kaminer J, Powers AS, Horn KG, Hui C, Evinger C (2011) Characterizing the spontaneous blink generator: an animal model. J Neurosci 31: 11256-11267.

4. Maffei A, Angrilli A (2018) Spontaneous eye blink rate: An index of dopaminergic component of sustained attention and fatigue. Int $\mathrm{J}$ Psychophysiol 123: 58-63.

5. Bentivoglio AR, Bressman SB, Cassetta E, Carretta D, Tonali P, et al. (1997) Analysis of blink rate patterns in normal subjects. MovDisord 12: 1028-1034.

6. Agostino R, Bologna M, Dinapoli L, Gregori B, Fabbrini G, et al. (2008) Voluntary, spontaneous, and reflex blinking in Parkinson's disease. MovDisord 23: 669-675.

7. Bologna M, Agostino R, Gregori B, Belvisi D, Ottaviani D, et al. (2009) Voluntary, spontaneous and reflex blinking in patients with clinically probable progressive supranuclear palsy. Brain 132: 502-510.

8. Bentivoglio AR, Daniele A, Albanese A, Tonali PA, Fasano A (2006) Analysis of blink rate in patients with blepharospasm. MovDisord 21: 1225-1229.
9. Ferrazzano G, Conte A, Belvisi D, Fabbrini A, Baione V, Berardelli A, et al. (2019) Writing, reading, and speaking in blepharospasm. J Neurol.

10. Feigin VL, Forouzanfar MH, Krishnamurthi R, Mensah GA, Connor M, et al. (2014) Global and regional burden of stroke during 1990-2010: findings from the Global Burden of Disease Study 2010. Lancet 383: 245255.

11. Dijkhuizen RM, Zaharchuk G, Otte WM (2014) Assessment and modulation of resting-state neural networks after stroke. CurrOpin Neurol 27: 637-643.

12. Anagnostou E, Kouzi I, Vassilopoulou S, Paraskevas GP, Spengos K (2012) Spontaneous eyeblink rate in focal cerebrovascular lesions.Eur Neurol. 67: 39-44.

13. Yoon HW, Chung JY, Song MS, Park H (2005) Neural correlates of eye blinking; improved by simultaneous fMRI and EOG measurement. Neurosci Lett 381: 26-30.

14. Planton S, Jucla M, Roux FE, Démonet JF (2013) The "handwriting brain": a meta-analysis of neuroimaging studies of motor versus orthographic processes. Cortex 49: 2772-2787.

15. Potgieser AR, van der Hoorn A, de Jong BM (2015) Cerebral activations related to writing and drawing with each hand. PLoS One 10: e 012672.

16. Pugh KR, Mencl WE, Jenner AR, Katz L, Frost SJ, et al. (2001) Neurobiological studies of reading and reading disability. J CommunDisord 34: 479-492.

17. Baker RS, Andersen AH, Morecraft RJ, Smith CD (2003) A functional magnetic resonance imaging study in patients with benign essential blepharospasm. J Neurophthalmol 23: 11-15. 